'Departamento de Ingeniería Industrial, Universidad de

Concepción. Concepción, Chile. angeniero Civil Industrial.

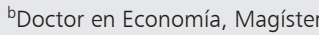
en Economía Ambiental y

Recursos Naturales.

Recibido el 12 de agosto de 2020, aceptado el 15 de abril de 2021.

Correspondencia a:

Cristian Mardones

Departamento de Ingeniería Industrial, Universidad de Concepción.

Edmundo Larenas 219. Concepción, Chile. crismardones@udec.cl

\section{Aporte de la gestión de episodios críticos a la reducción de enfermedades respiratorias en las ciudades de Los Ángeles y Chillán, Chile}

\author{
FELIPE HENRÍQUEZ ${ }^{1, \mathrm{a}}$, CRISTIAN MARDONES ${ }^{1, \mathrm{~b}}$
}

Background: In southern Chile cities, the emission of air pollutants, especially the use of firewood for heating is restricted during critical air pollution periods. Aim: To analyze how restrictions applied during the management of air pollution critical episodes have contributed to reduce emergency room admissions for respiratory diseases in two Chilean cities between 2013 and 2019. Material and Methods: Poisson regression models were estimated with daily data including explanatory variables, such as the daily and lag concentration of respirable particulate material $\left(P M_{10}\right)$, temperature, relative humidity, rainfall, wind speed, seasonal factors, and implementation of different types of critical episodes. Results: The implementation of restrictions during the management of critical pollution episodes decreased emergency room admissions for upper respiratory infections and bronchial obstructive crises, especially when an environmental emergency was decreed during the critical episode. However, the effect on each group of respiratory diseases was heterogeneous between cities, which could be related to avoidance behavior, indoor air pollution, the composition of $P M_{10}$ or the presence of other pollutants, and not just a reduction in the daily concentration of $P M_{10}$. Conclusions: The management of critical pollution episodes with restrictions to pollutant emissions is a useful measure to improve population health in cities that have implemented environmental decontamination plans.

(Rev Med Chile 2021; 149: 856-863)

Key words: Air Pollution; Particulate Matter; Respiratory Tract Diseases.

$\mathrm{L}$ as ciudades de Chillán y Los Ángeles registran altas concentraciones de material particulado respirable $\left(\mathrm{MP}_{10}\right)$ y fino $\left(\mathrm{MP}_{2,5}\right)$, superando las normas primarias de calidad ambiental establecidas en Chile. Esto es preocupante porque diversos estudios internacionales ${ }^{1-4} \mathrm{y}$ nacionales ${ }^{5-7}$ asocian altas concentraciones de material particulado con diversas enfermedades respiratorias.

Según el Ministerio del Medio Ambiente, la combustión de leña residencial fue responsable de $85,2 \%$ de las emisiones de $\mathrm{MP}_{2,5}$ a nivel nacional en el año 2017. Aunque este porcentaje es incluso mayor en algunas ciudades del centro-sur de Chile. Por ello, los planes de descontaminación implementados en las ciudades de Los Ángeles y Chillán han implementado la gestión de episodios críticos (GEC) para reducir la contaminación del aire en el corto plazo a través de restricciones temporales a las fuentes emisoras entre los meses 
de abril y septiembre. Específicamente, la GEC permite pronosticar las concentraciones con un día de anticipación y decretar un episodio crítico (alerta, pre-emergencia o emergencia ambiental) si se llegase a superar un cierto valor límite del Índice de Calidad del Aire por Partículas.

En el caso de decretarse una alerta ambiental se prohíbe el funcionamiento de calderas con una potencia térmica mayor a $75 \mathrm{kWt}$ que presenten emisiones de MP mayores a $50 \mu \mathrm{g} / \mathrm{m}^{3} \mathrm{~N}$ (no se explicita que sea solamente $\mathrm{MP}_{10}$, refiriéndose a la emisión continua de gases por el ducto), mientras que en el caso de una pre-emergencia o emergencia ambiental se prohíbe el funcionamiento de calderas industriales con emisiones de MP mayores a $30 \mu \mathrm{g} / \mathrm{m}^{3} \mathrm{~N}$. Adicionalmente, en un episodio de alerta o pre-emergencia se restringe el uso de leña para calefacción residencial, mientras que en un episodio de emergencia se prohíbe totalmente el uso de este combustible. Por otro lado, se prohíben las quemas de desechos agrícolas y/o forestales, y también, se restringen las actividades físicas en establecimientos educacionales durante cualquier episodio crítico.

La implementación de la GEC ha reducido significativamente las concentraciones de $\mathrm{MP}_{10}$ en Temuco, Chile ${ }^{8}$. Sin embargo, no hay evidencia de cómo la implementación de la GEC contribuye directamente a la reducción de enfermedades respiratorias. Cabe señalar que las ciudades de Los Ángeles y Chillán cuentan con un plan de descontaminación vigente y han implementado hace años la GEC, por lo cual es interesante determinar cuáles son las enfermedades respiratorias más afectadas por las concentraciones de $\mathrm{MP}_{10}$ y cuánto contribuye la GEC a disminuir las atenciones de urgencia en cada una estas enfermedades. Para llevar a cabo este estudio se utilizó una regresión Poisson que es un caso particular de los modelos lineales generalizados (GLM) $)^{9,10}$. Sin embargo, en la literatura también se han utilizado alternativas como el modelo quasi-Poisson ${ }^{11}$, el modelo binomial negativo ${ }^{12-14}$ o el modelo aditivo generalizado $(\mathrm{GAM})^{15,16}$.

\section{Material y Método}

\section{Datos}

Los datos diarios de atenciones de urgencia por enfermedades respiratorias fueron obtenidos desde el Departamento de Estadísticas e Información de la Salud de Chile (DEIS). Las atenciones de urgencia están clasificadas por causa según la Clasificación Estadística Internacional de Enfermedades y Problemas Relacionados con la Salud (CIE-10). Estos datos incluyen información de los Servicios de Urgencia de Alta Resolución (SAR), Servicios de Atención Primaria de Urgencia (SAPU) y hospitales. La Tabla 1 muestra que entre los años 2013 y 2019 la ciudad de Los Ângeles registró más atenciones de urgencia por enfermedades respiratorias que la ciudad de Chillán, y además, que la enfermedad respiratoria con más atenciones de urgencia fue IRA (infección respiratoria aguda) Alta, seguida por Bronquitis/ Bronquiolitis Aguda (BBA).

Los datos diarios de las concentraciones de $\mathrm{MP}_{10}$ se obtuvieron del Sistema de Información Nacional de Calidad del Aire (SINCA). También, existen registros diarios de $\mathrm{MP}_{2,5}$ pero hay menos datos continuos para este contaminante, por lo cual finalmente no fue incluido en el estudio. Además, se construyeron rezagos diarios de las concentraciones de $\mathrm{MP}_{10}$ considerando hasta cinco días para obtener el rezago promedio ya que diversos estudios ${ }^{9,12,14}$ muestran que existe una asociación entre las atenciones de urgencia por enfermedades respiratorias y la exposición a $\mathrm{MP}_{10}$ en los días previos a la atención.

Por otra parte, los registros históricos de episodios críticos se obtuvieron desde la Secretaria Regional del Ministerio del Medioambiente de la Región del Biobío (Tabla 2).

Los datos diarios de temperatura, humedad y velocidad del viento se obtuvieron del SINCA, los cuales se registraron en las mismas estaciones de monitoreo desde donde se obtuvieron las concentraciones de $\mathrm{MP}_{10}$. Por otro lado, las precipitaciones se obtuvieron de la Dirección General de Aguas (DGA).

Finalmente, se incluyeron variables que controlan la estacionalidad a través de tendencia lineal, cuadrática, sinusoidal, cosenoidal, feriados, día de la semana y mes.

\section{Modelo estadístico}

El número de pacientes ingresados a urgencias por enfermedades respiratorias en un día determinado es una variable de conteo. Para estimar modelos estadísticos con variable dependiente de conteo se utilizan frecuentemente los modelos 
Tabla 1. Resumen de atenciones de urgencia por enfermedades respiratorias

\begin{tabular}{|lcrcc|}
\hline Causa respiratoria & \multicolumn{2}{c}{ Los Ángeles } & \multicolumn{2}{c|}{ Chillán } \\
IRA alta (J00-J06) & $\begin{array}{c}\text { n del } \\
\text { atenciones }\end{array}$ & $\begin{array}{c}\text { n del } \\
\text { total }\end{array}$ & $\begin{array}{c}\text { atenciones } \\
\text { total }\end{array}$ \\
Influenza (J09-J11) & 385.953 & 61 & 248.716 & 65 \\
\hline Neumonía (J12-J18) & 7.969 & 1 & 6.508 & 2 \\
\hline Bronquitis/bronquiolitis aguda (BBA) (J20-J21) & 24.134 & 4 & 13.751 & 4 \\
\hline Crisis obstructiva bronquial (COB) (J40-J46) & 131.758 & 21 & 75.542 & 20 \\
\hline Otra causa respiratoria (OCR) (J22, J30-J39, J47, J60-J98) & 48.696 & 5 & 23.199 & 6 \\
\hline Total de causas respiratorias & 628.144 & 100 & 38.613 & 4 \\
\hline
\end{tabular}

Fuente: Elaboración propia.

Tabla 2. Episodios críticos por año

\begin{tabular}{|ccccccc|}
\hline Año & Alerta & $\begin{array}{c}\text { Los Ángeles } \\
\text { Pre-emergencia }\end{array}$ & Emergencia & Alerta & $\begin{array}{c}\text { Chillán } \\
\text { Pre-emergencia }\end{array}$ & Emergencia \\
2016 & 24 & 25 & 17 & 25 & 32 & 13 \\
2017 & 18 & 20 & 7 & 30 & 25 & 9 \\
2018 & 20 & 19 & 25 & 27 & 27 & 22 \\
2019 & 25 & 12 & 10 & 22 & 12 & 0 \\
\hline
\end{tabular}

Nota: Los datos de Chillán para 2019 fueron obtenidos desde Facebook (medio oficial de difusión). Fuente: SEREMI de Medio Ambiente de la Región del Biobío.

GLM. Uno de los GLM más usados es la regresión de Poisson ${ }^{17}$, la cual también se escogió para realizar este estudio. Utilizando el método de máxima verosimilitud es posible obtener los estimadores de los parámetros $\left(\beta_{k}\right)$ asociados a cada variable explicativa $x_{k}$.

No obstante, en la literatura epidemiológica se tiende a utilizar el riesgo relativo $(R R)$ para un incremento en la variable explicativa $x_{k}$, el cual se calcula a partir de la siguiente fórmula:

$$
R R=e^{\left(\beta_{k} \Delta x_{k}\right)}
$$

\section{Especificaciones de los modelos estadísticos}

Para confirmar la robustez de los resultados se estimaron distintas especificaciones de modelos Poisson por ciudad y por causa respiratoria. La primera especificación incluye todas las variables explicativas. La segunda especificación utiliza un criterio de eliminación de variables paso a paso (Stepwise Backward), el cual parte con un mo- delo que incluye todas las variables y de manera iterativa elimina variables del modelo según su significancia estadística (se eliminan variables con valor- $p>0,15)$. Finalmente, la tercera especificación utiliza un criterio de inclusión de variables paso a paso (Stepwise Forward), el cual parte con un modelo que incluye solo una constante y agrega variables al modelo de forma iterativa de acuerdo con su significancia estadística (se agregan variables con valor-p $<0,15)$.

\section{Resultados}

En esta sección solo se reportan los resultados obtenidos para las causas IRA Alta, crisis obstructiva bronquial (COB) y otra causa respiratoria (OCR), ya que el resto de las enfermedades respiratorias (Influenza, Neumonía y Bronquitis/Bronquiolitis Aguda: BBA) no presentan resultados robustos a distintas especificaciones de variables 
explicativas. Esto último, se explica porque todos los estudios epidemiológicos que utilizan datos de series de tiempo se basan en el supuesto que todas las variables relevantes están siendo incluidas en las regresiones, pero pueden existir otros factores no observables para el investigador que cambien a través del tiempo y que pueden afectar el número de atenciones de urgencia sesgando los resultados. En este estudio, esos factores no observables podrían ser cambios en la contaminación intradomiciliaria, proliferación de virus, composición del $\mathrm{MP}_{10}$, entre otros.

\section{Riesgo relativo para el $M P_{10}$}

Los resultados de $R R$ para un incremento de $1 \mu \mathrm{g} / \mathrm{m}^{3}$ de $\mathrm{MP}_{10}$ en las ciudades de Los Ángeles y Chillán se presentan en la Tabla 3. Para ejemplificar la interpretación del $R R$ se puede señalar que las atenciones de urgencia por IRA Alta se incrementarían entre $0,059 \%$ y $0,063 \%$ en la ciudad de Los Ángeles si el MP ${ }_{10}$ aumenta su concentración diaria en $1 \mu \mathrm{g} / \mathrm{m}^{3}$.

En ambas ciudades se observa que el aumento de las concentraciones diarias de $\mathrm{MP}_{10}$ contribuye a incrementar significativamente las atenciones de IRA Alta, COB y OCR. Los mayores riesgos relativos según enfermedad se obtienen para $\mathrm{COB}$ en la ciudad de Chillán. Sin embargo, el riesgo relativo para IRA alta es mayor en la ciudad de Los
Ángeles y el riesgo relativo para OCR es mayor en la ciudad de Chillán. Lo anterior, implica que la contaminación por $\mathrm{MP}_{10}$ tiene un efecto heterogéneo en la salud respiratoria de la población de cada ciudad. Estos resultados son robustos a la inclusión o exclusión de la variable de la concentración promedio del rezago de $\mathrm{MP}_{10}$ en los últimos cinco días, y también, a la especificación de los modelos estadísticos (Stepwise Backward, Stepwise Forward o todas las variables), excepto para las atenciones de urgencia por OCR.

Los resultados para las otras variables explicativas incluidas en los modelos estadísticos y no reportados por temas de espacio se discuten a continuación. El mayor impacto del $\mathrm{MP}_{10}$ se observa el mismo día de la atención de urgencia, pero la concentración promedio de los cinco días anteriores también tiene un impacto relevante en algunas enfermedades respiratorias. Las variables meteorológicas de temperatura, humedad y precipitaciones tienen signo negativo en la gran mayoría de los modelos, mientras que la velocidad del viento no tiene un efecto robusto. Lo anterior, es esperable ya que las bajas temperaturas afectan el sistema inmune, proliferación de virus, y también, el uso de calefacción a leña ${ }^{18,19}$. Cabe recordar que los virus se replican mejor a temperaturas frías que a la temperatura corporal ${ }^{20,21}$, lo cual se explica porque la temperatura de $37^{\circ} \mathrm{C}$ promueve la secre-

Tabla 3. Coeficientes de riesgo relativo para un incremento de $1 \mu \mathrm{g} / \mathrm{m}^{3}$ en la concentración diaria de $\mathrm{MP}_{10}$

\begin{tabular}{|c|c|c|c|c|c|}
\hline \multirow[b]{2}{*}{$\begin{array}{l}\text { Causa } \\
\text { respiratoria }\end{array}$} & \multirow[b]{2}{*}{$\begin{array}{l}\text { Inclusión de } \\
\text { variables }\end{array}$} & \multicolumn{2}{|c|}{ Los Ángeles } & \multicolumn{2}{|c|}{ Chillán } \\
\hline & & $\begin{array}{l}\text { Sin rezago } \\
\text { de } \mathrm{MP}_{10}\end{array}$ & $\begin{array}{l}\text { Con rezago } \\
\text { de } \mathrm{MP}_{10}\end{array}$ & $\begin{array}{l}\text { Sin rezago } \\
\text { de } \mathbf{M P}_{10}\end{array}$ & $\begin{array}{c}\text { Con rezago } \\
\text { de } \mathrm{MP}_{10}\end{array}$ \\
\hline IRA Alta & Todas & $1,00063^{* * *}$ & $1,00059 * * *$ & $1,00045^{* * *}$ & $1,00038^{* * *}$ \\
\hline IRA Alta & Stepwise Backward & $1,00063^{* * *}$ & $1,00059 * * *$ & $1,00046^{* * *}$ & $1,00039 * * *$ \\
\hline IRA Alta & Stepwise Forward & $1,00063^{* * *}$ & $1,00059 * * *$ & $1,00046^{* * *}$ & $1,00039^{* * *}$ \\
\hline $\mathrm{COB}$ & Todas & $1,00062 * *$ & $1,00062^{* *}$ & $1,00163^{* *}$ & $1,00138^{* *}$ \\
\hline $\mathrm{COB}$ & Stepwise Backward & 1,00071 ** & $1,00061 * *$ & $1,00164^{* *}$ & $1,00142^{* *}$ \\
\hline $\mathrm{COB}$ & Stepwise Forward & $1,00071 * *$ & $1,00060 * *$ & $1,00163^{* *}$ & $1,00140 * *$ \\
\hline OCR & Todas & 1,00029 & 1,00032 & 1,00107 & 1,00090 \\
\hline OCR & Stepwise Backward & $1,00041^{* *}$ & $1,00041 * *$ & $1,00108^{* *}$ & $1,00086^{* *}$ \\
\hline OCR & Stepwise Forward & 1,00041 ** & $1,00040 * *$ & $1,00107^{* *}$ & $1,00087^{* *}$ \\
\hline
\end{tabular}

Nota: *significativo al 5\%, **significativo al 1\% y ***significativo al 0,1\%; IRA Alta: Infección respiratoria aguda alta; COB: Crisis obstructiva bronquial; OCR: Otras causas respiratorias. Fuente: Elaboración propia. 
ción de interferones, pero incluso en ausencia de interferones, la temperatura más alta contribuye a un inicio más temprano de la muerte de las células infectadas por virus ${ }^{22}$. Por otro lado, las precipitaciones atrapan las partículas sólidas presentes en el aire lo que disminuye la concentración de $\mathrm{MP}_{10}{ }^{23,24}$, y además, en días de lluvia disminuye la actividad física y la interacción de las personas en espacios muy concurridos. La velocidad del viento debería mejorar la circulación del aire ${ }^{23,25}$, pero no se observa un efecto claro sobre las atenciones de urgencia en las enfermedades respiratorias analizadas.

Las variables estacionales asociadas a día de la semana y mes tienen un comportamiento casi idéntico en todas las especificaciones de modelos estadísticos. Por ejemplo, febrero es el único mes del año que tiene un impacto negativo, los meses de otoño-invierno (entre marzo y septiembre) tienen el mayor número de atenciones de urgencia en los diferentes tipos de enfermedades respiratorias estudiadas ${ }^{26}$.

\section{Implementación de restricciones por la GEC}

En la Tabla 4 se presenta el efecto de decretar una alerta, pre-emergencia y emergencia ambiental sobre el número de atenciones de urgencia en las ciudades de Los Ángeles y Chillán.

Los resultados para la ciudad de Los Ángeles revelan que decretar alerta ambiental reduce significativamente el número de atenciones de urgencia por IRA Alta (entre 4,0\% y 4,7\%), pero no reduce significativamente las atenciones de urgencia por $\mathrm{COB}$ u OCR. En el caso de decretar pre-emergencia ambiental se reduce significativamente el número de atenciones de urgencia por IRA Alta (entre 3,5\% y 4,7\%) y OCR (entre 11,9\% y $12,9 \%$ ), pero no se reducen significativamente las atenciones de urgencia por COB. A su vez, decretar emergencia ambiental reduce significativamente el número de atenciones de urgencia por IRA Alta (entre $6,6 \%$ y $8,1 \%$ ) y OCR (entre $28,4 \%$ y $30,6 \%$ ), pero no reduce significativamente las atenciones de urgencia por COB. Por otra parte, los resultados para la ciudad de Chillán muestran que decretar alerta ambiental solamente genera una reducción significativa y robusta en el número de atenciones de urgencia por COB (entre 14,0\% y $18,2 \%)$. En el caso de decretar pre-emergencia ambiental solo se reduce significativamente el número de atenciones de urgencia por $\mathrm{COB}$ (entre
$14,2 \%$ y $19,9 \%)$. No obstante, decretar emergencia ambiental reduce significativamente el número de atenciones de urgencia por IRA Alta (entre 4,1\% y $4,9 \%$ ), COB (entre $19,4 \%$ y $25,3 \%$ ), y también, OCR (entre $14,6 \%$ y $17,1 \%$ ). Cabe señalar que las enfermedades agrupadas en el término OCR incluyen: Infección aguda no especificada de las vías respiratorias inferiores; Otras enfermedades de las vías respiratorias superiores; Bronquiectasia; Enfermedades del pulmón debidas a agentes externos; Otras enfermedades respiratorias que afectan principalmente al intersticio; Afecciones supurativas y necróticas de las vías respiratorias inferiores; Derrame pleural no clasificado en otra parte; Derrame pleural en afecciones clasificadas en otra parte; Paquipleuritis; Neumotórax; Otras afecciones de la pleura; Trastornos del sistema respiratorio consecutivos a procedimientos no clasificados en otra parte; Insuficiencia respiratoria no clasificada en otra parte; y Otros trastornos respiratorios.

\section{Discusión}

El estudio originalmente consideró enfermedades como Influenza, Neumonía y Bronquitis/ Bronquiolitis aguda, pero no se obtuvieron resultados concluyentes, por lo cual solo se reportan los efectos para las atenciones de urgencia por IRA Alta, COB y OCR.

Los resultados muestran que un aumento en la concentración del $\mathrm{MP}_{10}$ genera un aumento significativo y robusto en las atenciones de urgencia por IRA Alta, COB y OCR. Por otra parte, la implementación de la GEC ha contribuido a reducir significativamente las atenciones de urgencia de estas enfermedades respiratorias. Las atenciones de urgencia por OCR han tenido la mayor reducción porcentual en la ciudad de Los Ángeles, mientras que las atenciones de urgencia por COB han tenido la mayor reducción porcentual en la ciudad de Chillán. Esta variabilidad en los impactos se puede atribuir a que decretar un episodio crítico no solo reduce la contaminación por $\mathrm{MP}_{10}$, sino que también genera un efecto de evitación que reduce el deseo de asistir a los servicios de urgencia para evitar exponerse a contagios adicionales o a altos niveles de contaminación. Para testear este efecto evitación se estimaron los impactos sobre las atenciones de urgencia por diarrea aguda ya que 


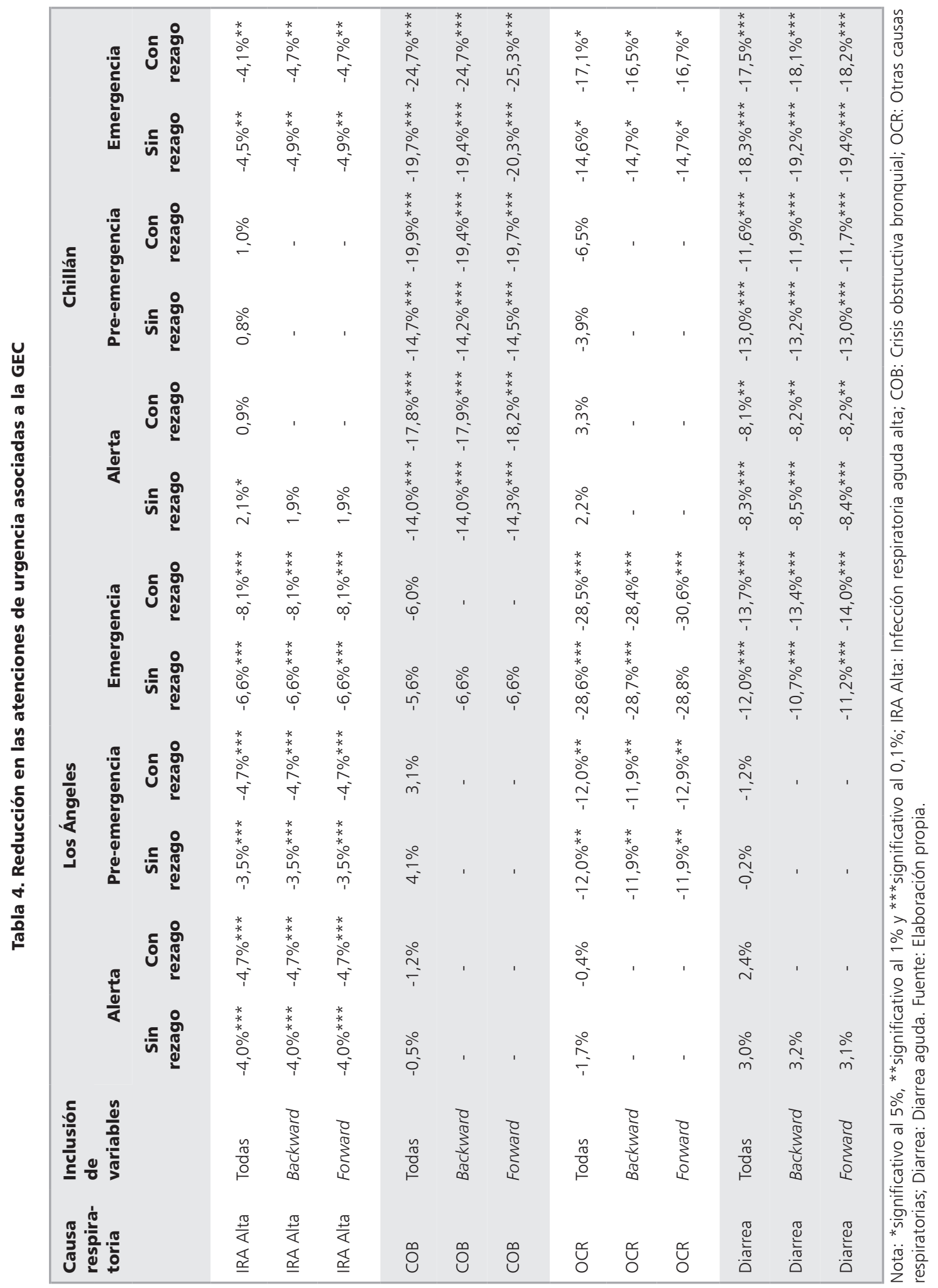


no existe una relación causal del $\mathrm{MP}_{10}$ con este tipo de enfermedad, demostrándose que efectivamente no existe un efecto estadísticamente significativo con respecto a las concentraciones de este contaminante, pero hay una reducción significativa durante los episodios de emergencia ambiental en Los Ángeles y durante los episodios de alerta, pre-emergencia y emergencia en Chillán (Tabla 4).

La mayor reducción en el número de atenciones respiratorias de urgencia por decretar emergencia ambiental es esperable ya que en este tipo de episodio crítico son más intensas las restricciones para las fuentes emisoras de contaminantes y actividades al aire libre. Sin embargo, llama la atención que el impacto sobre algunas enfermedades respiratorias es diferente según la ciudad bajo estudio. Lo anterior, podría atribuirse a un efecto de evitación para no exponerse a enfermedades respiratorias en centros de salud y no solamente a la reducción efectiva de la contaminación. Una explicación alternativa para los diferentes resultados es el rol de la contaminación intradomiciliaria en ambas comunas, pero no existen datos disponibles en Chile para controlar este factor. Tampoco, se solucionaría levantando encuestas ya que el enfoque metodológico realizado en el presente estudio requiere el uso de datos diarios. Otra potencial explicación sería que la composición del $\mathrm{MP}_{10}$ fuera diferente entre ciudades. Cabe señalar que los antecedentes para formular los planes de descontaminación en ambas ciudades demuestran que la utilización de leña para calefacción residencial es la principal fuente emisora en Chillán (86\%) y Los Ángeles (86\%), aunque las emisiones de otros contaminantes como $\mathrm{NO}_{\mathrm{x}}$ y $\mathrm{SO}_{2}$ son mucho mayores en Chillán.

Así, se concluye que la implementación de GEC ha contribuido sustancialmente a reducir el número de atenciones de urgencia en algunas enfermedades respiratorias: IRA alta y COB, especialmente cuando se decreta episodio de emergencia ambiental.

\section{Referencias}

1. Pope III C, Coleman N, Pond Z, Burnett R. Fine particulate air pollution and human mortality: $25+$ years of cohort studies. Environ Res 2020; 183: 108924.

2. Chen C, Hsieh Y, Su H, Wu J. Causality test of ambient fine particles and human influenza in Taiwan: Age group-specific disparity and geographic heterogeneity. Environ Int 2018; 111: 354-61.

3. Szyszkowicz M, Kousha T, Castner J, Dales R. Air pollution and emergency department visits for respiratory diseases: A multi-city case crossover study. Environ Res 2018; 163: 263-9.

4. Davidson C, Phalen R, Solomon P. Airborne Particulate Matter and Human Health: A Review. Aerosol Sci Tec 2005; 39: 737-49.

5. Prieto-Parra L, Yohannessen K, Brea C, Vidal D, Ubilla C A, Ruiz-Rudolph P. Air pollution, PM2.5 composition, source factors, and respiratory symptoms in asthmatic and nonasthmatic children in Santiago, Chile. Environ Int 2017; 101: 190-200.

6. Ruiz-Rudolph P, Arias N, Pardo S, Meyer M, Mesías S, Galleguillos C, et al. Impact of large industrial emission sources on mortality and morbidity in Chile: a sma11-areas study. Environ Int 2016; 92: 130-8.

7. Mardones C, Saavedra A, Jiménez J. Health and economic benefits of reducing $10 \mu \mathrm{m}$ particulate matter $\left(\mathrm{PM}_{10}\right)$ in Metropolitan Area of Concepción, Chile. Rev Med Chile 2015; 143: 475-83.

8. Mardones C, Cornejo N. Ex-post evaluation of a program to reduce critical episodes due to air pollution in Southern Chile. Environ Impact Asses 2020; 80: 106334.

9. Wang Y, Shi Z, Shen F, Sun J, Huang L, Zhang H, et al. Associations of daily mortality with short-term exposure to PM2.5 and its constituents in Shanghai, China. Chemosphere 2019; 233: 879-87.

10. Li G, Xue M, Zeng Q, Cai Y, Pan X, Meng Q. Association between fine ambient particulate matter and daily total mortality: An analysis from 160 communities of China. Sci Total Environ 2017; 599-600: 108-13.

11. Rhee J, Dominici F, Zanobetti A, Schwartz J, Wang Y, Di Q, et al. Impact of Long-Term Exposures to Ambient PM2.5 and Ozone on ARDS Risk for Older Adults in the United States. Chest 2019; 156: 71-9.

12. Ardiles L, Tadano Y, Costa S, Urbina V, Capucim M, Da Silva I, et al. Negative Binomial regression model for analysis of the relationship between hospitalization and air pollution. Atmos Pollut Res 2018; 9: 333-41.

13. Nayebare S, Aburizaiza O, Siddique A, Carpenter D, Pope C, Mirza H, et al. Fine particles exposure and cardiopulmonary morbidity in Jeddah: A time-series analysis. Sci Total Environ 2019; 647: 1314-22.

14. Carugno M, Dentali F, Mathieu G, Fontanella A, Mariani J, Bordini L, et al. PM10 exposure is associated with increased hospitalizations for respiratory syncytial virus bronchiolitis among infants in Lombardy, Italy. Environ Res 2018; 166: 452-57.

15. Chi R, Li H, Wang Q, Zhai Q, Wang D, Wu M, et al. 
Association of emergency room visits for respiratory diseases with sources of ambient PM2.5. J Environ Sci 2019; 86: 154-63.

16. Zuo B, Liu C, Chen R, Kan H, Sun J, Zhao J, et al. Associations between short-term exposure to fine particulate matter and acute exacerbation of asthma in Yancheng, China. Chemosphere 2019; 237: 124497.

17. Wooldridge JM. Econometric analysis of cross section and panel data. 2nd ed. Cambridge, Mass: MIT Press; 2010.

18. Xu X, González J, Shen S, Miao S, Dou J. Impacts of urbanization and air pollution on building energy demands-Beijing case study. Appl Energy 2018; 225: 98-109.

19. Schueftan A, Sommerhoff J, González A. Firewood demand and energy policy in south-central Chile. Energy Sustain Dev 2016; 33: 26-35.

20. Bel G, Holst, M. Evaluation of the impact of Bus Rapid Transit on air pollution in Mexico City. Transp Policy, 2018; 63: 209-20.

21. Tyrrell DA, Parsons R. Some virus isolations from common colds. III. Cytopathic effects in tissue cultures. Lancet, 1960; 1(7118): 239-242.

22. Turner R, Couch R. Rhinoviruses. Fields Virology, eds Knipe DM, Howley, PM (Lippincott, Williams, and Wilkins, Philadelphia) 2007; 1: 895-909.

23. Foxman E, Storer J, Vanaja K, Levchenko A, Iwasaki A. Temperature-dependent control of common cold virus. PNAS, 2016; 113 (30): 8496-501.

24. Karagiannidis A, Poupkou A, Giannaros T, Giannaros C, Melas D, Argiriou A. The air quality of a mediterranean urban environment area and its relation to major meteorological parameters. Water Air Soil Pollut 2015; 226(1): 2239.

25. Clements N, Hannigan M, Miller S, Peel J, Milford J. Comparisons of urban and rural PM10-2.5 and PM2.5 mass concentrations and semi-volatile fractions in northeastern Colorado. Atmos Chem Phys 2016; 16: 7469-84.

26. Moriyama M, Hugentobler WJ, Iwasaki A. Seasonality of Respiratory Viral Infections. Annu Rev Virol 2020; 7(1): 83-101. 